\title{
Variational integrators for degenerate Lagrangians, with application to point vortices
}

\author{
Clarence W. Rowley \\ Princeton University \\ cwrowley@princeton.edu
}

\author{
Jerrold E. Marsden \\ California Institute of Technology \\ marsden@cds.caltech.edu
}

\begin{abstract}
We develop discrete mechanics and variational integrators for a class of degenerate Lagrangian systems, and apply these integrators to a system of point vortices. Excellent numerical behavior is observed. A longer term goal is to use these integration methods in the context of control of mechanical systems, such as coordinated groups of underwater vehicles. In fact, numerical evidence given in related problems, such as those in [2] shows that in the presence of external forces, these methods give superior predictions of energy behavior.
\end{abstract}

\section{Introduction}

Variational integration methods preserve key system properties at the discrete level. There are discrete versions of the canonical nature of the the time evolution maps, discrete versions of associated conservation laws, etc. See [4] for a survey and references. Variational algorithms get their name from the fact that it is the variational principles of mechanics that are discretized rather than the equations. They extend to forced (e.g., by control actuation) and dissipative systems. They give (see [2]) superior predictions about energy conservation for conservative systems and energy change for forced and dissipative systems over standard (even more highly accurate) schemes. Symplectic integration methods for point vortices were given in [7], which clearly shows their superiority. However, the methods were somewhat hand crafted and special. In this paper, we do these simulations by general methods and numbers of vortices, getting even better results.

The usual theory of variational integrators and discrete mechanics for integration of Lagrangian systems (see [4]) assumes that the Lagrangian is regu- lar. However, some potential applications, such as a system of interacting point vortices have degenerate Lagrangians (see [5]). Our main theoretical contribution deals with degenerate Lagrangians in the context of discrete mechanics and variational integrators. We consider a particular class of degenerate Lagrangians, namely those that are affine in the velocities. For an application, we consider point vortices in the plane and demonstrate the excellent numerical properties of the algorithms. It would be of interest to also develop them for point vortices on the sphere and we expect the techniques here to also apply to that situation; see [6] for an interesting, but different approach.

\section{Background on discrete mechanics}

Given a configuration space $Q$, and Lagrangian $L: T Q \rightarrow \mathbb{R}$, Hamilton's principle, that solutions $q(t) \in Q$ are critical points of the action integral

$$
S=\int_{a}^{b} L(q(t), \dot{q}(t)) d t
$$

(with fixed endpoints $q(a)$ and $q(b)$ ), leads to the Euler-Lagrange equations

$$
\frac{d}{d t} \frac{\partial L}{\partial \dot{q}}-\frac{\partial L}{\partial q}=0
$$

Discrete mechanics begins with a discrete $L a$ grangian $L_{d}: Q \times Q \rightarrow \mathbb{R}$, normally obtained from $L$ by approximating (1) along solutions:

$$
L_{d}\left(q_{0}, q_{1}\right) \approx \int_{0}^{h} L(q(t), \dot{q}(t)) d t,
$$

where $q(0)=q_{0}$ and $q(h)=q_{1}$, and $h$ is the timestep. Given $L_{d}$, the discrete Hamilton's principle extremizes the action sum

$$
S_{d}=\sum_{k=0}^{N-1} L_{d}\left(q_{k}, q_{k+1}\right)
$$


over $q_{1}, \ldots, q_{N-1}$, given fixed endpoints $q_{0}$ and $q_{N}$. This discrete variational principle leads to the discrete Euler-Lagrange equations

$$
D_{1} L_{d}\left(q_{k+1}, q_{k+2}\right)+D_{2} L_{d}\left(q_{k}, q_{k+1}\right)=0
$$

for all $k=1, \ldots, N-1$, where $D_{1} L_{d}$ denotes the derivative of $L_{d}$ with respect to its first slot. This equation implicitly defines a discrete algorithm

$$
\Phi:\left(q_{k}, q_{k+1}\right) \mapsto\left(q_{k+1}, q_{k+2}\right): Q \times Q \rightarrow Q \times Q,
$$

which satisfies

$$
D_{1} L_{d} \circ \Phi+D_{2} L_{d}=0 .
$$

The algorithm $\Phi$ is well defined if the map $D_{1} L_{d}$ : $Q \times Q \rightarrow T^{*} Q$ is invertible (usually guaranteed by the implicit function theorem for pairs $\left(q_{0}, q_{1}\right)$ of nearby points and small time steps).

\subsection{Symplectic structure}

A symplectic structure on a manifold $M$ is a nondegenerate closed two-form $\Omega$, and a diffeomorphism $\varphi: M \rightarrow M$ is called symplectic if it preserves this symplectic structure: that is, if $\varphi^{*} \Omega=\Omega$. The typical way one shows that Lagrangian flows are symplectic is by introducing the fiber derivative

$$
\mathbb{F} L: T Q \rightarrow T^{*} Q:\left(q^{i}, \dot{q}^{i}\right) \mapsto\left(q^{i}, \frac{\partial L}{\partial \dot{q}^{i}}\right),
$$

and defining the two-form $\Omega_{L}$ on $T Q$ by pulling back the canonical form $\Omega$ on $T^{*} Q$ : that is, $\Omega_{L}=$ $(\mathbb{F} L)^{*} \Omega$. In coordinates, this form is given by

$$
\Omega_{L}=\frac{\partial^{2} L}{\partial \dot{q}^{i} \partial q^{j}} \mathbf{d} q^{i} \wedge \mathbf{d} q^{j}+\frac{\partial^{2} L}{\partial \dot{q}^{i} \partial \dot{q}^{j}} \mathbf{d} q^{i} \wedge \mathbf{d} \dot{q}^{j},
$$

which is nondegenerate if and only if the matrix $\partial^{2} L / \partial \dot{q}^{i} \partial \dot{q}^{j}$ is nonsingular. In this case, $L$ is regular. Otherwise, $L$ is degenerate. If $L$ is regular, then (2) are second order, and the flow map $\varphi_{t}: T Q \rightarrow T Q$ is symplectic (i.e., $\varphi_{t}^{*} \Omega_{L}=\Omega_{L}$ ).

However, if $L$ is degenerate, then the two-form $\Omega_{L}$ is degenerate, and it does not even make sense to ask whether the flow on $T Q$ is symplectic (though one may still study whether $\Omega_{L}$ is preserved by the flow map, as long as the flow is well defined). In this paper, we address a special class of degenerate Lagrangians for which a symplectic structure is well defined. For the cases we address, however, the symplectic structure will not be defined on $T Q$, but rather on $Q$.

\subsection{Variational integrators are symplectic} Just as the flow of the Euler-Lagrange equations is symplectic if $L$ is regular, one can show that the algorithm $\Phi$ determined by the discrete EulerLagrange equations is symplectic. One defines the discrete fiber derivative

$$
\mathbb{F} L_{d}: Q \times Q \rightarrow T^{*} Q:\left(q_{0}, q_{1}\right) \mapsto\left(q_{1}, D_{2} L\left(q_{0}, q_{1}\right)\right)
$$

and, as in the continuous case, one defines the discrete Lagrangian two-form $\Omega_{L_{d}}$ by $\Omega_{L_{d}}=$ $\left(\mathbb{F} L_{d}\right)^{*} \Omega$, where again $\Omega$ is the canonical form on $T^{*} Q$. In coordinates, $\Omega_{L_{d}}$ has the form

$$
\Omega_{L_{d}}=\frac{\partial^{2} L_{d}}{\partial q_{0}^{i} \partial q_{1}^{i}} \mathbf{d} q_{0}^{i} \wedge \mathbf{d} q_{1}^{j}
$$

As before, this form is nondegenerate if and only if the matrix $\partial^{2} L_{d} / \partial q_{0}^{i} \partial q_{1}^{j}$ is nonsingular, and in this case we say that the discrete Lagrangian is regular. Note that the discrete Lagrangian must be regular in order for the algorithm $\Phi$ to be well defined. Just as in the continuous case, one can show (see, for example, [4]) that the algorithm $\Phi$ determined by (3) exactly preserves the symplectic form $\Omega_{L_{d}}$ (i.e., $\Phi^{*} \Omega_{L_{d}}=\Omega_{L_{d}}$ ).

\section{Degenerate Lagrangian systems}

In this section, we describe the class of degenerate Lagrangians we address, motivated by the example of point vortices interacting in a twodimensional potential flow.

\subsection{Example: point vortices}

Consider a system of $n$ interacting point vortices in two dimensions, where the coordinates of the $j$ th vortex in the complex plane are given by $z^{j}=$ $x^{j}+i y^{j}$. If the circulation of the $j$ th vortex is $\Gamma_{j}$, then the Lagrangian is given by (see, e.g., [5])

$$
\begin{aligned}
L\left(z^{j}, \bar{z}^{j}, \dot{z}^{j}, \dot{\bar{z}}^{j}\right) & =\frac{1}{2 i} \sum_{j=1}^{n} \Gamma_{j}\left(\bar{z}^{j} \dot{z}^{j}-z^{j} \dot{\bar{z}}^{j}\right) \\
& -\frac{1}{2 \pi} \sum_{j \neq k}^{n} \Gamma_{j} \Gamma_{k} \log \left|z^{j}-z^{k}\right|,
\end{aligned}
$$


or in real form,

$$
\begin{aligned}
& L(x, y, \dot{x}, \dot{y})=\frac{1}{2} \sum_{j=1}^{n} \Gamma_{j}\left(x^{j} \dot{y}^{j}-y^{j} \dot{x}^{j}\right) \\
&-\frac{1}{4 \pi} \sum_{j \neq k}^{n} \Gamma_{j} \Gamma_{k} \log \left(\left(x^{j}-x^{k}\right)^{2}+\left(y^{j}-y^{k}\right)^{2}\right) .
\end{aligned}
$$

Though this Lagrangian is degenerate, Hamilton's principle still applies, and the Euler-Lagrange equations give the first-order evolution equations

$$
\dot{\bar{z}}^{j}=\frac{1}{2 \pi i} \sum_{j \neq k} \frac{\Gamma_{k}}{z^{j}-z^{k}} .
$$

The above Lagrangian has the general form

$$
L(q, \dot{q})=\langle\alpha(q), \dot{q}\rangle-H(q),
$$

where $\alpha$ is a one-form on $Q$, and $H$ is a function on $Q$. For the point vortex Lagrangian (4), with $q=(x, y) \in \mathbb{R}^{2 n}$, the form $\alpha$ is given in coordinates by

$$
\alpha\left(x^{i}, y^{i}\right)=-\frac{1}{2} \Gamma_{i j} y^{i} d x^{j}+\frac{1}{2} \Gamma_{i j} x^{i} d y^{j},
$$

where $\Gamma_{i j}=\Gamma_{i} \delta_{i j}$ is a diagonal matrix of vortex strengths. It is well known (see, e.g., [5]) that this point vortex system possesses a Hamiltonian (symplectic) structure, and the motivation for the present work is to obtain integrators which preserve this symplectic structure.

\subsection{Hamiltonian structure}

We now investigate the equations of motion that arise from Lagrangians of the form (6), and the sense in which these equations are Hamiltonian. Note that there are other classes of degenerate Lagrangians [1], but the form (6) arises in many physical examples, including not just point vortices, but also quantum mechanics! For these systems, the momentum is given by

$$
p_{i}=\frac{\partial L}{\partial \dot{q}^{i}}=\alpha_{i}(q),
$$

and so the Euler-Lagrange equations become

$$
\frac{d}{d t}\left(\alpha_{i}(q)\right)=\frac{\partial L}{\partial q^{i}}=\frac{\partial \alpha_{j}}{\partial q^{i}} \dot{q}^{j}-\frac{\partial H}{\partial q^{i}}
$$

and hence

$$
\frac{\partial \alpha_{i}}{\partial q^{j}} \dot{q}^{j}-\frac{\partial \alpha_{j}}{\partial q^{i}} \dot{q}^{j}=-\frac{\partial H}{\partial q^{i}} .
$$

These are first-order equations of the form

$$
\dot{q}=X(q)
$$

where the vector field $X$ is given by

$$
-\mathbf{d} \alpha(X(q), v)=\mathbf{d} H(q) \cdot v
$$

for all $v \in T_{q} Q$. This equation shows that the equations of motion (8) are Hamiltonian, where the symplectic two-form is given by $\Omega=-\mathbf{d} \alpha$. Denoting the flow of $X$ by $\varphi_{t}: Q \rightarrow Q$, it is straightforward to show that $\varphi_{t}$ is a symplectic transformation (i.e., $\varphi_{t}^{*} \Omega=\Omega$ ), and the Hamiltonian $H$ is preserved by the flow (i.e., $H=H \circ \varphi_{t}$ ).

For the point vortex Lagrangian (4), the symplectic form is therefore

$$
\Omega=-\mathbf{d} \alpha=-\Gamma_{i j} d x^{i} \wedge d y^{j} .
$$

It is important to note the differences between the usual case for regular Lagrangians, and the degenerate case discussed here. For regular Lagrangians, the Euler-Lagrange equations produce second-order equations, while for degenerate Lagrangians of the form (6), they give the first-order equations (8). Furthermore, in the regular case, the symplectic structure is a two-form on $T^{*} Q$ or, on the Lagrangian side, on $T Q$, while here the symplectic structure is a two-form on $Q$. This distinction will be important when we discuss the sense in which the integrators we derive are symplectic.

\subsection{The action and symplectic structures}

Define the action integral

$$
S(q, t)=\int_{0}^{t} L(q(s), \dot{q}(s)) d s,
$$

where $q(s)$ denotes the solution to the EulerLagrange equations with $q(0)=q$. Recall that in the degenerate case being considered here, the Euler-Lagrange equations are first order. Define $S_{t}: Q \rightarrow \mathbb{R}$ by $S_{t}(q)=S(q, t)$. In this formulation, we regard $q(t)$ as a function of the initial condition $q$. Thus, for example, the differential $\mathbf{d} q$ at time $t$ is obtained by using the first variation of the equations (i.e., the tangent $T F_{t}$ of the flow map $\left.F_{t}: Q \rightarrow Q\right)$. This induces a first variation of the curve, and hence the velocity $\dot{q}$, which can 
be used in the variational principle. Then taking the exterior derivative, we have

$$
\begin{aligned}
\mathbf{d} S_{t} & =\mathbf{d} \int_{0}^{t} L\left(q^{i}, \dot{q}^{i}\right) d t \\
& =\int_{0}^{t}\left(\frac{\partial L}{\partial q^{i}} d q^{i}+\frac{\partial L}{\partial \dot{q}^{i}} d \dot{q}^{i}\right) d t \\
& =\int_{0}^{t}\left(\frac{\partial L}{\partial q^{i}}-\frac{d}{d t} \frac{\partial L}{\partial \dot{q}^{i}}\right) d q^{i} d t+\left.\frac{\partial L}{\partial \dot{q}^{i}} d q^{i}\right|_{0} ^{t} \\
& =\left.\alpha_{i} d q^{i}\right|_{0} ^{t}=F_{t}^{*} \alpha-\alpha
\end{aligned}
$$

since $d q^{i}$ at time $t$ is obtained from that at time $t=0$ by the tangent to the flow map, as explained above. (See [3], §8.2 for the corresponding calculation for regular Lagrangians). Thus, since $\mathbf{d}^{2} S_{t}=0$, we obtain

$$
F_{t}^{*} \mathbf{d} \alpha-\mathbf{d} \alpha=0
$$

which is one way of seeing that the symplectic form $\Omega=-\mathbf{d} \alpha$ is preserved by the flow map.

\section{Discrete mechanics for degenerate Lagrangians}

\subsection{Variational integrators}

One choice of the discrete Lagrangian is

$$
L_{d}\left(q_{0}, q_{1}\right)=h \cdot L\left((1-\sigma) q_{0}+\sigma q_{1}, \frac{q_{1}-q_{0}}{h}\right) .
$$

Writing $q_{k+\sigma}=(1-\sigma) q_{k}+\sigma q_{k+1}$, the discrete Euler-Lagrange equations become

$$
\begin{gathered}
\sigma \frac{\partial \alpha_{j}}{\partial q^{i}}\left(q_{k+\sigma}\right) \frac{q_{k+1}^{j}-q_{k}^{j}}{h}+(1-\sigma) \frac{\partial \alpha_{j}}{\partial q^{i}}\left(q_{k+1+\sigma}\right) \frac{q_{k+2}^{j}-q_{k+1}^{j}}{h} \\
-\frac{\alpha_{i}\left(q_{k+1+\sigma}\right)-\alpha_{i}\left(q_{k+\sigma}\right)}{h}=\sigma \frac{\partial H}{\partial q^{i}}\left(q_{k+\sigma}\right)+(1-\sigma) \frac{\partial H}{\partial q^{i}}\left(q_{k+1+\sigma}\right) .
\end{gathered}
$$

For point vortices, this expression simplifies considerably:

$$
\frac{\bar{z}_{k+2}^{j}-\bar{z}_{k}^{j}}{2 h}=\frac{1}{2 \pi i} \sum_{j \neq l} \Gamma_{l}\left(\frac{\sigma}{z_{k+\sigma}^{j}-z_{k+\sigma}^{l}}+\frac{1-\sigma}{z_{k+1+\sigma}^{j}-z_{k+1+\sigma}^{l}}\right) .
$$

It is insightful to compare this expression to the form of the continuous evolution equations (5). If $f(z)$ denotes the complex conjugate of the right hand side of (5), then these equations have the form

$$
\frac{z_{k+2}-z_{k}}{2 h}=\sigma f\left(z_{k+\sigma}\right)+(1-\sigma) f\left(z_{k+1+\sigma}\right) .
$$

For $\sigma=0$ or $\sigma=1$, the scheme is explicit, and is just the leapfrog scheme, which is often used in molecular dynamics simulations where exact energy conservation is critical. For $\sigma \in(0,1)$ it gives a (possibly new) family of implicit schemes. The structure of the scheme is illustrated in Figure 1.

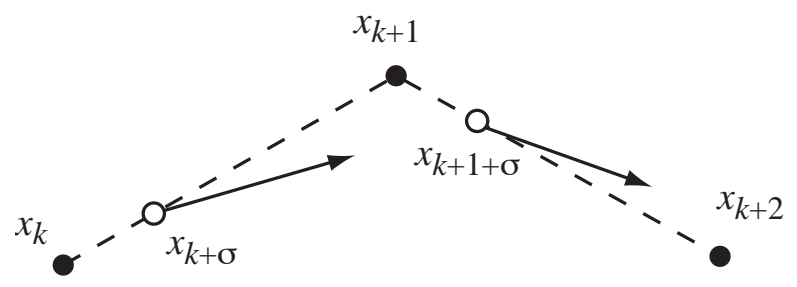

Figure 1: Structure of the scheme (14) for point vortices: Closed circles indicate final values of $x$, arrows indicate evaluations of the right hand side $f(x)$, and open circles indicate temporary values of $x$ used for function evaluation.

Accuracy and stability. To assess the accuracy stability of the method, we consider applying the scheme (14) to the linear equation $\dot{x}=\lambda x$. The integration scheme (14) is formally secondorder accurate (the truncation error is $O\left(h^{3}\right)$ ) for all values of $\sigma$ except $\sigma=(3-\sqrt{3}) / 6$, for which the scheme is fourth-order accurate. A standard stability analysis shows that the scheme is stable only when $\lambda$ is purely imaginary, with

$$
|\operatorname{Im}(\lambda h)|<\frac{1}{|1-2 \sigma|} .
$$

For $\sigma=1 / 2$, the region of stability is the entire (closed) left half plane.

Thus, which scheme to use depends on a tradeoff between speed, accuracy, and stability. The scheme with $\sigma=0$ is explicit, so requires the least computation per timestep, but has the most severe timestep restriction of the family, $|\lambda h|<$ 1. The implicit scheme with $\sigma=1 / 2$ has no timestep restriction, but the implicit scheme with $\sigma=(3-\sqrt{3}) / 2$ is the most accurate.

\subsection{Discrete symplectic structure}

Let us look at the discrete symplectic structure that is conserved by our family of variational integrators. The discrete symplectic structure $\Omega_{L_{d}}$ is a two-form on $Q \times Q$, given by

$$
\Omega_{L_{d}}=\frac{\partial^{2} L_{d}}{\partial q_{0}^{i} \partial q_{1}^{j}} \mathbf{d} q_{0}^{i} \wedge \mathbf{d} q_{1}^{j} .
$$


In coordinates, our discrete Lagrangian is given by

$$
L_{d}\left(q_{0}^{i}, q_{1}^{i}\right)=\alpha_{i}\left(q_{\sigma}\right)\left(q_{1}^{i}-q_{0}^{i}\right)-h H\left(q_{\sigma}\right)
$$

where $q_{\sigma}=(1-\sigma) q_{0}+\sigma q_{1}$. Therefore, one computes

$$
\begin{aligned}
\frac{\partial^{2} L_{d}}{\partial q_{0}^{i} \partial q_{1}^{j}} & =h \sigma(1-\sigma) \frac{\partial^{2} \alpha_{k}}{\partial q^{i} \partial q^{j}}\left(q_{\sigma}\right) \frac{q_{1}^{k}-q_{0}^{k}}{h} \\
& -\sigma \frac{\partial \alpha_{i}}{\partial q^{j}}\left(q_{\sigma}\right)+(1-\sigma) \frac{\partial \alpha_{j}}{\partial q^{i}}\left(q_{\sigma}\right) \\
& -h \sigma(1-\sigma) \frac{\partial^{2} H}{\partial q^{i} \partial q^{j}}\left(q_{\sigma}\right) .
\end{aligned}
$$

This is the form that is preserved by the integrator. It is not clear precisely when this form is nondegenerate, as one reviewer pointed out. One might hope that this form is nondegenerate (on $Q \times Q$ ) whenever $\mathbf{d} \alpha$ is nondegenerate (on $Q$ ), but this is unfortunately not the case, and in fact the degeneracy of $\Omega_{L_{d}}$ depends on the choice of $\alpha$, not just $\mathbf{d} \alpha$. However, for the point vortex example, with $\alpha$ given by (7), $\Omega_{L_{d}}$ is nondegenerate, at least for small time steps.

One easily checks that this form is close to the continuous symplectic form $\Omega=-\mathbf{d} \alpha$ : in the limit as $h \rightarrow 0$ (and $q_{1} \rightarrow q_{0}$ ), the first and third lines of (17) vanish, and the form (15) becomes

$$
\Omega_{L_{d}} \approx\left(\frac{\partial \alpha_{j}}{\partial q_{i}}-\sigma\left(\frac{\partial \alpha_{i}}{\partial q_{j}}+\frac{\partial \alpha_{j}}{\partial q_{i}}\right)\right) \mathbf{d} q_{0}^{i} \wedge \mathbf{d} q_{1}^{j} .
$$

The term that multiplies $\sigma$ will cancel (it is symmetric), and we are left with, for $q_{0}-q_{1}=O(h)$,

$$
\Omega_{L_{d}} \approx \frac{\partial \alpha_{j}}{\partial q_{i}} \mathbf{d} q^{i} \wedge \mathbf{d} q^{j}=\mathbf{d} \alpha,
$$

which is precisely the continuous symplectic form from section 3.2.

The fact that the continuous symplectic form is in the above sense, close to the discrete form, and the discrete form is exactly preserved, means that numerically, the continuous form will remain close to its exact value indefinitely for all bounded motions.

\section{Numerical results: point vortices}

We performed several numerical experiments to evaluate the performance of the symplectic integrators, relative to some standard integrators of comparable accuracy. The schemes considered are as follows:

\begin{tabular}{clcc} 
Scheme & Name & Order & Type \\
\hline RK2 & Runge-Kutta & 2 & Explicit \\
RK4 & Runge-Kutta & 4 & Explicit \\
VE2 & Variational, $\sigma=0$ & 2 & Explicit \\
VI2 & Variational, $\sigma=0.5$ & 2 & Implicit
\end{tabular}

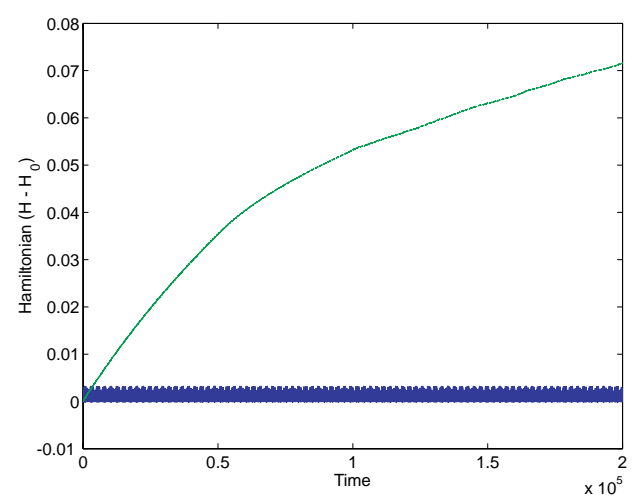

Figure 2: Hamiltonian $(H(t)-H(0))$ for scheme RK2 (dashed) and symplectic scheme VE2 (solid).

Explicit scheme. Figure 2 shows the variation in the Hamiltonian for schemes VE2 and RK2 for a system of four point vortices, with the following initial configuration:

\begin{tabular}{c|rrrr}
$j$ & 1 & 2 & 3 & 4 \\
\hline$x^{j}$ & -1 & 1 & -1 & 1 \\
$y^{j}$ & 2 & 2 & -2 & -2 \\
$\Gamma_{j}$ & 1 & 1 & -1 & -1
\end{tabular}

These initial conditions are symmetric about the line $y=0$, and as time evolves, the two pairs of vortices leapfrog past each other, as shown in Figure 3. Both simulations are performed for time $0 \leq t \leq 2 \times 10^{5}$, with a timestep $h=2$ for scheme VE2 and $h=1$ for scheme RK2 (the simulation using scheme RK2 blows up if the larger timestep $h=2$ is used). The variational scheme VE2 is not self-starting, so scheme RK2 was used for the first timestep. Because scheme RK2 requires two right-hand-side evaluations for each timestep, the computational time for scheme RK2 is approximately four times that of scheme VE2. 


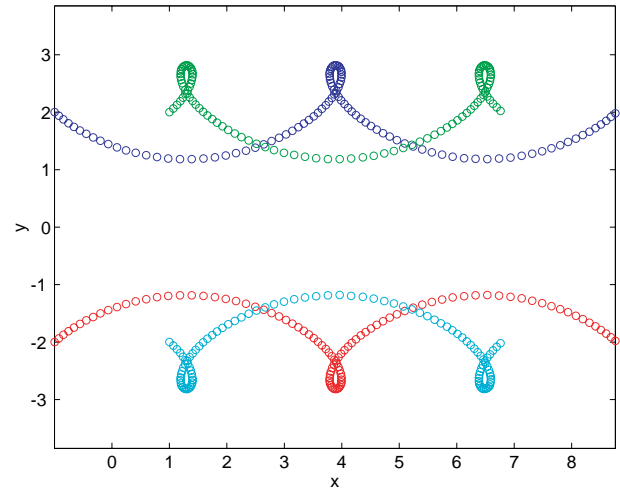

Figure 3: Typical trajectories of leapfrogging vortices for numerical tests.

From Figure 2, it is apparent that the scheme RK2 shows a secular drift in the Hamiltonian, while for the symplectic scheme VE2, the Hamiltonian remains close to its initial value for all time.

Implicit scheme. To evaluate the performance of an implicit variational integrator $(\sigma=1 / 2)$, we used the following two sets of initial conditions, also used by [7]:

\begin{tabular}{c|rrrr}
$j$ & 1 & 2 & 3 & 4 \\
\hline$x^{j}$ & 1.0 & 0.866 & -0.85 & -0.5 \\
$y^{j}$ & 1.0 & 0.45 & -1.363636 & -0.15 \\
\hline \hline$x^{j}$ & -1.85 & -0.07 & 0.78 & 0.76 \\
$y^{j}$ & 0.05 & -1.332 & 0.167 & -1.2902
\end{tabular}

For the first case, the motion is quasi-periodic, and for the second case, the motion is chaotic. Poincaré sections of the two cases are shown in Figures 4 and 5 , in which the results from schemes VI2 and RK4 are compared. The section shown is for $R_{2}=0, d R_{2} / d t>0$, and the $\left(R_{1}, P_{1}\right)$-plane is plotted, where the coordinates $R_{j}, P_{j}$ are as defined in [7]. The simulations in Figure 4 were carried out for $0 \leq t \leq 10^{5}$, and for Figure 5 for $0 \leq t \leq 10^{6}$.

Both schemes yield accurate results if a small enough timestep is used. However, as the timestep is increased, the Poincaré sections for scheme RK4 degrade much sooner than those for scheme VI2, even though RK4 is a higher-order scheme. For all of these cases, the behavior of the Hamiltonian is qualitatively similar to that shown in Figure 2:
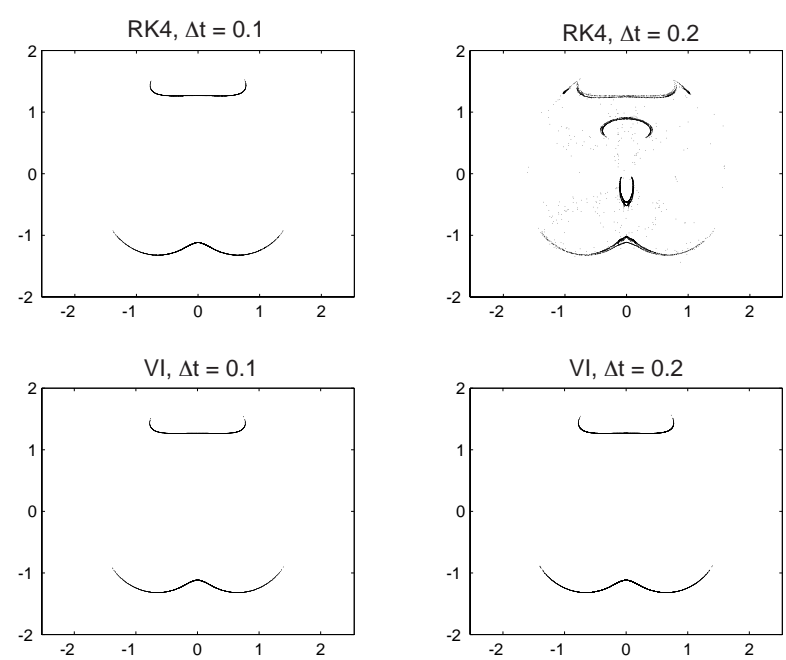

Figure 4: Poincaré map: quasiperiodic solutions. Both schemes give qualitatively correct results for $h=0.1$, but scheme RK4 gives spurious results for $h=0.2$.
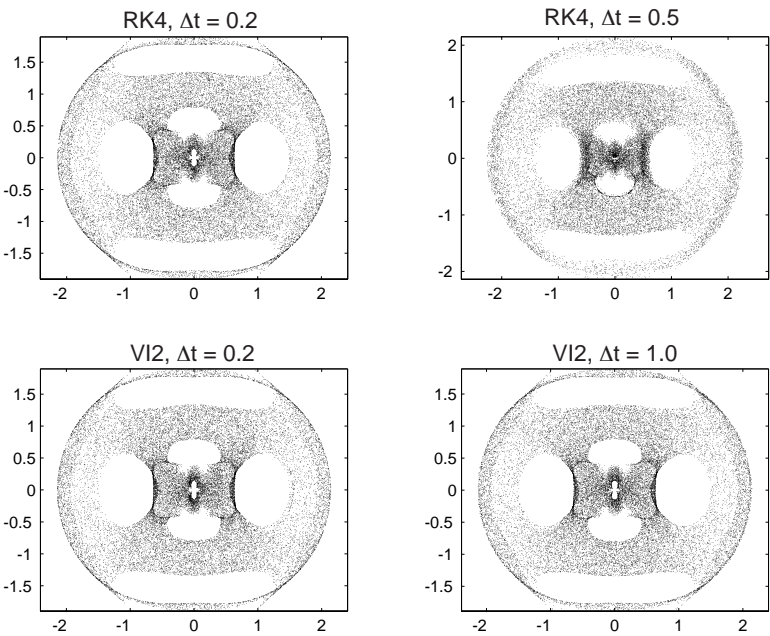

Figure 5: Poincaré map: chaotic solutions. Both schemes produce clear Poincaré sections for $h=0.2$, but for $h=0.5$, scheme RK4 produces a blurred section, while the section from scheme VI2 remains crisp for $h=1.0$. For $h=1.0$, scheme RK4 deviates completely, and transitions into a quasiperiodic state, as in Figure 4. 
scheme VI2 stays close to its original value, while scheme RK4 drifts.

\section{Conclusions}

We have developed the theory of variational integrators for a class of degenerate Lagrangians and have applied it to the case of point vortices in the plane to produce symplectic and momentum preserving schemes for arbitrary numbers of vortices. We have demonstrated with some numerical examples, that these schemes have excellent energy preserving properties compared to standard schemes and capture the subtle chaotic dynamics in a superior way.

\section{References}

[1] Cantrijn, F., J. F. Cariñena, M. Crampin, L. A. Ibort [1986], Reduction of degenerate Lagrangian systems. J. Geom. Phys. 3(3), 353-400.

[2] Kane, C, J. E. Marsden, M. Ortiz and M. West [2000], Variational integrators and the Newmark algorithm for conservative and dissipative mechanical systems, Int. J. Num. Math. Eng., 49, 1295-1325.

[3] Marsden, J. E. and T. S. Ratiu [1999], Introduction to Mechanics and Symmetry, volume 17 of Texts in Applied Mathematics, vol. 17; 1994, Second Edition, 1999. Springer-Verlag.

[4] Marsden, J. E. and M. West [2001], Discrete mechanics and variational integrators, Acta Numerica 10, $357-514$.

[5] Newton, P. K. [2001], The N-vortex Problem: Analytical techniques. Springer-Verlag, New York.

[6] Newton, P. K. and B. Khushalani [2002], Integrable decomposition methods and ensemble averaging for non-integrable $N$-vortex problems (preprint).

[7] Pullin, D. I. and P. G. Saffman [1991], Long time symplectic integration: the example of four-vortex motion, Proc. Roy. Soc. Lon. A 432, 481-494. 